\title{
The Impact of Cancer in the Tigray Region of Ethiopia: A Secondary Analysis
}

Hadgu Gerensea ${ }^{*}$, Mebrahtu Abay ${ }^{2}$, Hafte Teklay ${ }^{3}$, Zeray Baraki', Lidya Tsegay ${ }^{1}$ Teklit Angesom², Desta Siyoum ${ }^{1}$, Eskedar Brhane ${ }^{1}$, Teklit Grum $^{2}$, Solomon Hintsa ${ }^{2}$, Awoke Woldu' ${ }^{1}$, Almaz Sied ${ }^{4}$, and Patricia Malloy ${ }^{5}$

${ }^{1}$ School of Nursing, College of Health Science and Referral Hospital, Aksum University, Aksum, Ethiopia

${ }^{2}$ School of Public Health, College of Health Science and Referral Hospital, Aksum University, Aksum, Ethiopia

${ }^{3}$ School of Bio-medical, College of Health Science and Referral Hospital, Aksum University, Aksum, Ethiopia

${ }^{4}$ Tikur Anbessa Specialized Hospital, Addis Ababa, Ethiopia

${ }^{5}$ College of Nursing, University of Saskatchewan, Canada (North America)

\begin{abstract}
Control of communicable diseases has been the focus in the Tigray region. The research team, all of whom live in this region, propose that non-communicable diseases need to have this same control. To comprehend the impact of cancer, a non-communicable disease, the team conducted a secondary analysis of all patients who had received care in the Tigray region over a 4-year period. Over 10,000 registrations were reviewed; descriptive statistics provided the team with a rough estimate of the impact of cancer in this region. What follows is a more descriptive illustration of the impact with recommendations to decision makers in the Tigray region.
\end{abstract}

\section{Keywords: Cancer; Tigray region; Ethiopia}

Abbreviation: TASH: Tikur Anbessa Specialized Hospital; US: United State; HPV: Human Papilloma Virus; HIMS: Health Information Management System; IPD: Inpatient Department; OPD: Out Patient Department; IRB: Institution Review Board; TRHB: Tigray Region Health Bureau

\section{Introduction}

The Tigray region is the most northern region of Ethiopia, the capital Me'eklle is $782 \mathrm{~km}$ north of Addis Ababa, the nation's capital. The majority of the Tigray region population reside in rural areas and income is limited which leads to limited education opportunities for the people. Location, remote and rural nature of the Tigray region, coupled with education needs to be fully comprehended to understand the impact of a cancer diagnosis. Ethiopia due to little public awareness that cancer can be cured there has been little public demand that health systems address cancer [1]. Public health approach that identifies what can be done to diagnose and treat cancers more effectively at each level of the health system in low- and middle-income countries will require addressing the cancer epidemic [2,3]. Because of limited resources and other pressing public health problems, including communicable diseases such as acquired immune deficiency syndrome (AIDS)/human immunodeficiency virus (HIV) infection, malaria, and tuberculosis; cancer continues to receive low public health priority in Africa despite this growing burden [4]. The belief of "cancer cannot be cured" has persisted even into the $21^{\text {st }}$ century to some extent which has served to feel the fear people have of the disease [5]. Cancer managements are given very less attention. Globally, deaths associated with a cancer diagnosis is projected to increase with it doubling in a 20 year span (2000 to 2020). In low to middle income countries this rate is projected to jump 5 times over the same time period [6]. By 2050, 75\% of all deaths in low in countries is projected to be associated with a cancer diagnosis. Contrast this to the combined deaths associated with AIDS, tuberculosis, and malaria and cancer remains the victor [7]. In 2008, 12.7 million new cases of cancer with 7.6 million deaths [7].

Moreover, there is a debate in the trend of cancer regarding its exact magnitude and mortality. Due to inconsistencies in both research methods and registries, health professionals and policy makers are challenged in decision making regarding cancer hence, this would help as an input so.

Similarly, in the absence of reliable population registration in developing countries provide the only available window to observe the disease pattern in the community through all governmental health centers and hospital registration or statistics.

\section{Secondary Analysis: Methods and Results}

The Tigray region has 18 public hospitals and 170 health centers with a total population of 4,316,988. All patients who sought care from August 2011 to August 2015 in the public hospitals and Woreda health offices were included in the analysis. No data was excluded since all were complete and readable. Data was collected from the HMIS registration. A total of 10118 cancer diagnosis from inpatient and outpatient departments was extracted. More than $50 \%$ of the patients were female and approximately $6.5 \%$ were less than 4 years of age (Table 1). The diagnosis confirmation by fine needle aspiration cytology (FNAC), bone marrow (BM) aspiration, ultrasound, $\mathrm{x}$-ray and computed tomography (CT) scan. First, the data was entered and coded to Epi info version 3.5.4 and exported to analyses into SPSS version 21 window 7. Descriptive statistics provided the authors with an image of the patient, the type of cancer. This information contributed to the discussion on the impact of cancer in the Tigray region. Breast cancer is the most common cancer $(32.33 \%)$ from in patient department (IPD). The diagnosis of cancer increased annually. The magnitude of

*Corresponding authors: Hadgu Gerensea, School of Nursing, College of Health Science and Referral Hospital, Aksum University, Aksum, Ethiopia, Tel: 251914388918; E-mail: hadguellen1@gmail.com

Received February 18, 2017; Accepted March 31, 2017; Published April 03, 2017

Citation: Gerensea H, Abay M, Teklay H, Baraki Z, Tsegay L, et al. (2017) The Impact of Cancer in the Tigray Region of Ethiopia: A Secondary Analysis. J Cancer Sci Ther 9: 389-392. doi: 10.4172/1948-5956.1000447

Copyright: (c) 2017 Gerensea H, et al. This is an open-access article distributed under the terms of the Creative Commons Attribution License, which permits unrestricted use, distribution, and reproduction in any medium, provided the original author and source are credited. 
Citation: Gerensea H, Abay M, Teklay H, Baraki Z, Tsegay L, et al. (2017) The Impact of Cancer in the Tigray Region of Ethiopia: A Secondary Analysis. J Cancer Sci Ther 9: 389-392. doi: 10.4172/1948-5956.1000447

cancer morbidity in $2014 / 15$ is 2.12 times higher than $2011 / 12$ and the magnitude of mortality is more than double.

Furthermore, the magnitude of cancer in outpatient department (OPD) is increasing every year except in $2013 / 14$ which is more or less similar with $2012 / 13$. The $2014 / 15$ magnitude of cancer is 2.9 times higher than 2011/12 (Figures 1-3).

\section{Discussion}

Even though there is no full data and enough evidence of diagnosis of cancer registry in Ethiopia yet, starting from 2011/12 HMIS was introduced. There is also deficient registration of death certificates and an underestimation of cancer mortality in Ethiopia. In the absence of reliable population registration in developing countries like Ethiopia using HMIS is the only available window to observe the trend and pattern of cancer morbidity and mortality in Tigray region.

The study shows breast cancer is the leading causes of cancer

\begin{tabular}{|c|c|c|c|}
\hline \multirow{2}{*}{ Age in Year } & \multicolumn{2}{|c|}{ Sex } & Total Number \\
\cline { 2 - 4 } & Male Number (\%) & Female Number (\%) & $(\%)$ \\
\hline$<4$ & $383(43.27)$ & $289(56.73)$ & $672(6.6)$ \\
\hline$>4$ and $<15$ & $439(49.6)$ & $446(50.4)$ & $885(8.75)$ \\
\hline$>15$ & $3332(38.9)$ & $5229(61.1)$ & $8561(84.65)$ \\
\hline Total & $4154(41.1)$ & $5964(58.9)$ & 10118 \\
\hline
\end{tabular}

Table 1: Age and sex of participants from 2011-2015 in Tigray region. morbidity in the Tigray region. Its trend is also increasing every year. This is similar to study from Gondar showing that increased frequency over time [8]. Furthermore, in spite of awareness and screening female breast cancer has continued to show an increasing trend in Panama $[4,9]$.

Not only in morbidity, but also the breast cancer is leading cause of death among of all types of cancer with increasing trend every year. In contrast to this finding, breast cancer mortality rates have been declining among women in many western countries since the 1970s $[10,11]$. This difference may be related to government health policy since chronic diseases are neglected due to the massive burden of infectious diseases [12].

The commonest cancer seen in this study is breast cancer followed by unspecified malignant neoplasm and lymphoma unlike study in TASH which shows leukemia, lymphoma. This may be related to the difference of study participants since the study seen in TASH was only on pediatric [13]. Unspecified malignant neoplasm is the second most common seen in this study unlike study from lesser developed countries with cancer of stomach and cervix [14].

This difference may be related to variation in diagnostic procedures and quality of registration since the registration in Tigray, Ethiopia is unspecified.

Globally by 2020, the number of cancer morbidity and mortality will

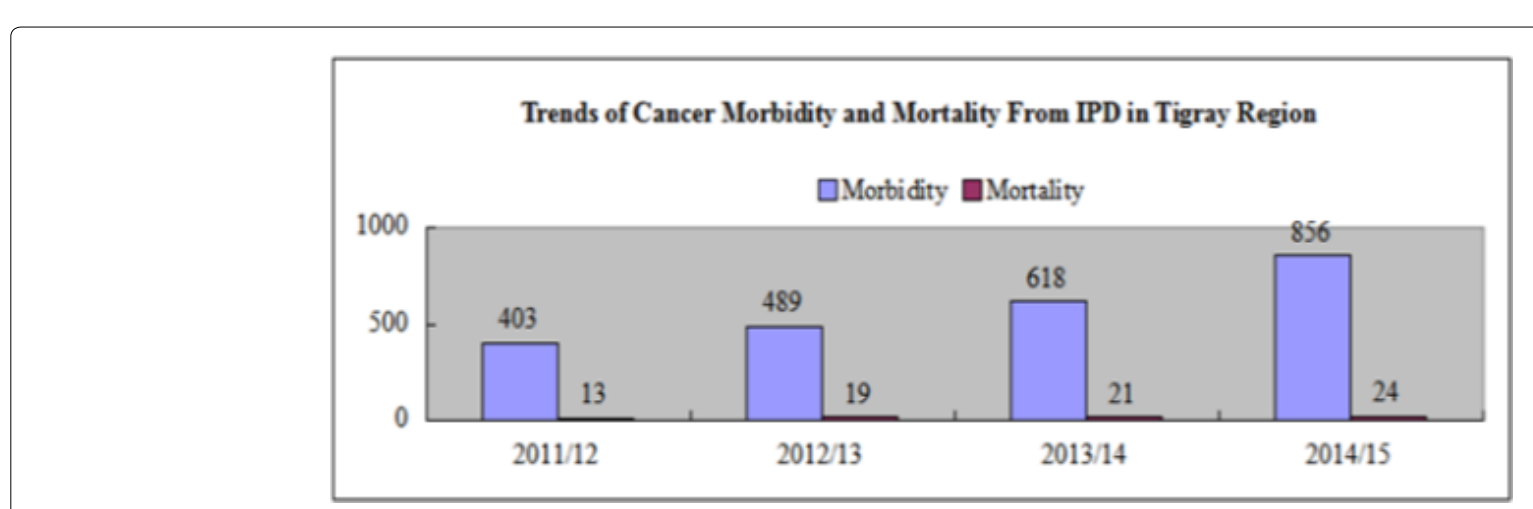

Figure 1: Trend of cancer morbidity and mortality in Tigray region.

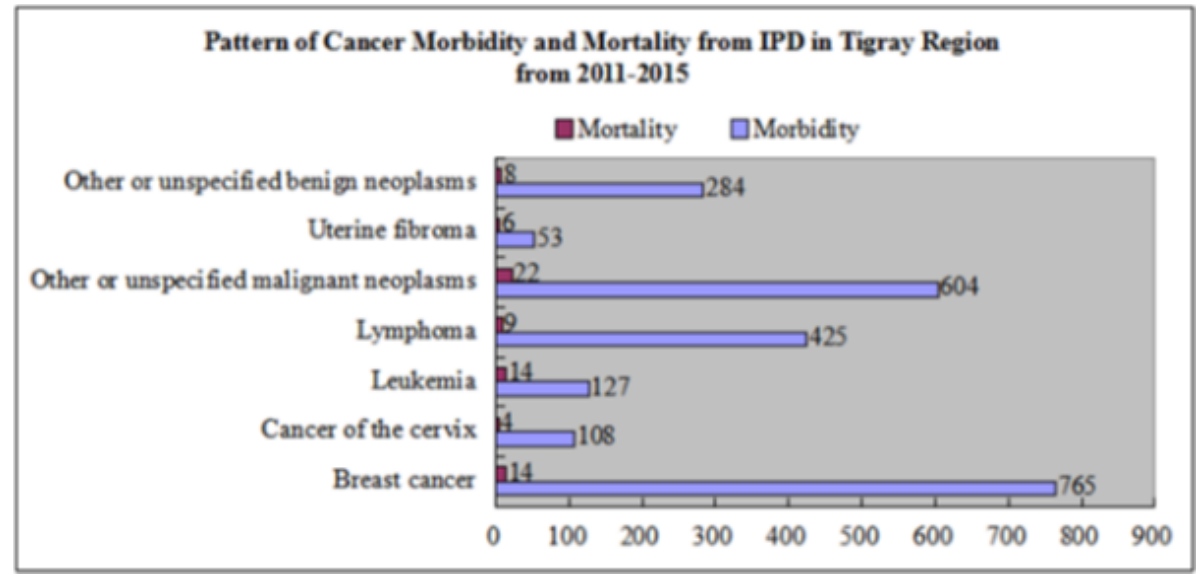

Figure 2: Pattern of cancer morbidity and mortality in Tigray region. 


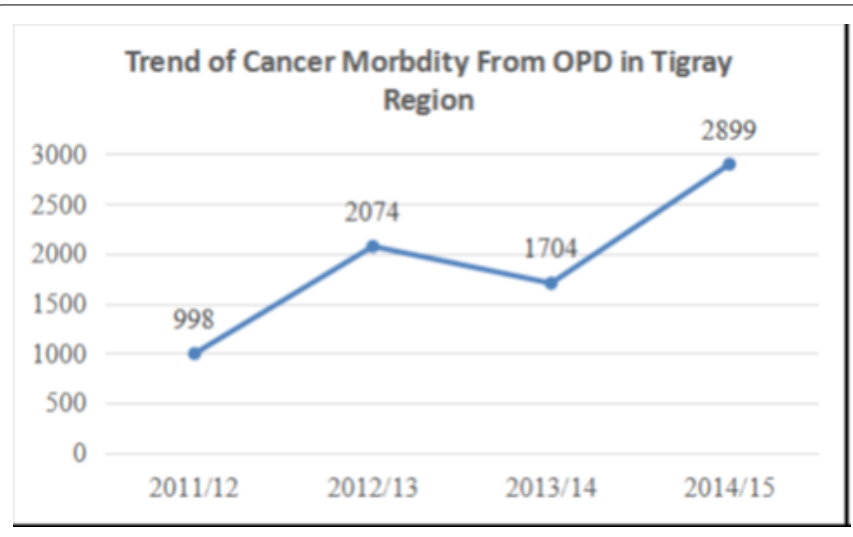

Figure 3: Trend of cancer at OPD in Tigray region.

increase with and the burden will greater in developing countries [4]. The finding of this research also suggested there would be an alarming increment in the coming years since within four-year, morbidity and mortality are increasing more than triple and double respectively.

This figure is supported by this research as the mortality 2.12 times within four years from 2011/12-2014/15. This finding is also supported by another research finding which shows three-quarters of all cancer deaths will be in low-income countries by $2050[2,3]$.

Similarly, in 2012, almost 1.1 million new cancer cases were estimated annually $[13,14]$. This is also supported by this finding. The finding of this study is analogous both to the reports from global estimation (51,000 per year) and from records of TASH radiotherapy center (120,500 per year) [14].

Furthermore, study in Spain and US showed that rapidly declined in breast cancer mortality. A similar finding is also observed in Argentina and Chile [15]. This difference may be related with due to accurate diagnosis followed by effective treatment exists in highincome countries.

Studies from Mexico and Colombia indicate relatively stable rates unlike these from Brazil and Chile which is high [16], but higher in this study since Ethiopia lacks trained medical personnel, adequate facilities and sufficient supply of essential chemotherapy drugs.

Another study from another East African nation, Tanzania shows similar finding. Since both countries are at similar level of development and health policy (focuses on infectious than the non-infectious disease) [17].

Despite increasing trend of cervical cancer morbidity and mortality, it is less common compared to another pattern of cancer. But a study from Panama indicates the 2nd most common next to breast cancer in women [16]. This difference is related to the characteristic of study participants. The trend of cervical cancer morbidity and mortality also decrease in Panama and some Latin American countries like Chile and Uruguay $[15,16]$, unlike this study. This difference may be related to economic status and Ethiopia focuses on Infectious disease. Similarly, some countries already are offering HPV vaccine through public immunization programs $[18,19]$. But other Latin American countries have seen comparable increasing in mortality trends $[15,18]$.

\section{Recommendations}

The trend of cancer morbidity and mortality in Tigray region is increasing by 2.12 and 2 times from inpatient department respectively. Similarly, the morbidity of cancer is increasing 3 times from outpatient department.

Overall cancer trend is increasing with breast cancer as the first leading cause of morbidity and mortality followed by the unspecified malignant neoplasm. Generally, contrary to common misperception, the burden of cancer morbidity and mortality will be worst in the coming few years as their increment are alarming and cancers are not recognized as a major illness due to several other competing causes of death like HIV/AIDS and TB. Even though there is no need of complete paradigm shift from communicable to non-communicable diseases like Cancer, Policy makers and NGO should focus on that neglected disease.

Even though, there is no need of complete paradigm shift from communicable to non-communicable diseases like Cancer, Policy makers and NGO should focus on those neglected diseases.

\section{Declarations}

Ethical approval and consent to participate: Institution review board (IRB) of Aksum University, College of Health Science has reviewed the protocol to ensured full protection of the rights of study subjects. Following the approval by IRB of Aksum University, IRB of Tigray Region Health bureau also approved and an official letter of co-operation was written to the directorate of health information system. In order to keep confidentiality of any information obtained, the data collection procedure was treated anonymously. Since it is secondary data direct participant of humans and animals are not involved.

Consent for publication: All Author and co-authors are agreed on the publication of the research on Omics Cancer Science and Therapy.

Competing interest: There is no competing interest

Funding: There is no funding

\section{Acknowledgment}

We would like to thanks to Tigray Region Health Bureau for giving the data.

\section{References}

1. Clinical Cancer Advances (2009) Major research advances in cancer treatment prevention, and screening. American Society of Clinical Oncology: J Clin Onco 27(35): 6052-6069.

2. Lodge M, Corbex M (2011) The evidence base for cancer control in developing countries: What is to be done? The newsletter of the international network for cancer treatment and research. The Breast 2: S65-69.

3. Benjamin O, Michel B, Colin B (2006) Access to cancer treatment in low- and middle-income countries.

4. Ferlay J, Shin HR, Bray F, Forman D, Mathers CD (2010) Cancer incidence and mortality worldwide: IARC Cancer Base No.10. Lyon, France: International Agency for Research on Cancer.

5. Davies SM (2007) Subsequent malignant neoplasm's in survivors of childhood cancer: Childhood cancer survivor study (CCSS) studies. Pediatr Blood Cancer 48: $727-730$.

6. Garcia M (2007) Global cancer facts and figures. American Cancer Society Atlanta, Georgia.

7. Ibekwe CM (2009) Factors influencing cervical cancer screening uptake among women attending Mahalapye District Hospital in Botswana-Use of the Health Belief Model.

8. Yifru S, Muluye D (2015) Childhood cancer in Gondar University Hospital, Northwest Ethiopia, BMC Res Notes 8: 474.

9. Gonzalez-Robledo MC, Gonzalez-Robledo LM, Nigenda G (2013) Public policy-making on breast cancer in Latin America. Rev Panam Salud Publica 33: 183-189.

10. Jemal A, Chu KC, Tarone RE (2001) Recent trends in lung cancer mortality in the United States. JNCI J Nation Cancer Inst 93(4): 277-283.

11. $\mathrm{NCl}$ Changing patterns in survival for U.S. women with invasive breast cancer (2015).

12. Aziza S, Julia C, Mary LC (2013) Pediatric oncology in Ethiopia: An IINCTR- 
Citation: Gerensea H, Abay M, Teklay H, Baraki Z, Tsegay L, et al. (2017) The Impact of Cancer in the Tigray Region of Ethiopia: A Secondary Analysis. J Cancer Sci Ther 9: 389-392. doi: 10.4172/1948-5956.1000447

USA and Georgetown University hospital twinning initiative with Tikur an bessa specialized hospital. cancer control: 108-112.

13. Franco EL, Tsu V, Herrero R, Lazcano-Ponce E, Hildesheim A, et al. (2008) Integration of human papillomavirus vaccination and cervical cancer screening in Latin America and the Caribbean. Vaccine 26(11): L88-L95.

14. Magrath I, Steliarova-Foucher E, Sidnei E, Ribeiro RC, Harif M, et al. (2013) Pediatric cancer in low-income and middle-income countries. Lancet Oncol 14: e104-e106.

15. Vidal C, Hoffmeister L, Biagini L (2013) Trend in cervical cancer mortality in Chile: Application of joinpoint regression models. Rev Panam Salud Publica 33: $407-413$
16. Politis M, Higuera G, Chang LR, Gomez B, Bares J, et al. (2015) Trend analysis of cancer mortality and incidence in Panama, using joinpoint regression analysis. Medicine (Baltimore) 94: e970.

17. Ribeiro RC, Steliarova-Foucher E, Magrath I, Lemerle J, Eden T, et al. (2008) Baseline status of paediatric oncology care in ten low-income or mid-income countries receiving my child matters support: A descriptive study. Lancet Oncol 9: 721-729.

18. Bosetti C (2205) Trends in cancer mortality in the Americas, 1970-2000. Ann Oncol 16: 489-511.

19. Herrero R, Gonzalez P, Markowitz LE (2015) Present status of human papillomavirus vaccine development and implementation. Lancet Oncol 16(5) e206-e216. 